Aus der Kgl, dermatologischen Universitätsklinik zu Breslau.

\title{
Zur Kenntnis der diphtherischen Hautentzündungen, besonders der durch echte Diphtheriebazillen hervorgerufenen.
}

\author{
Von \\ Dr. Arthur Schucht, \\ Spezialarzt für Hautkrankheiten in Danzig, früherer Assistent der Klinik. \\ (Hiezu Taf. VII.)
}

In den neueren Lehr- und Handbüchern der Dermatologie ist die Diphtherie der Haut zumeist mit Stillschweigen übergangen worden, oder es sind ihrer Besprechung nur wenige Zeilen gewidmet. W. Scholtz nennt die primäre Diphtherie der Haut eine seltene Erkrankung, und so weit es sich nicht um Ulzerationen der äußeren Haut handelt, die gleichzeitig mit Schleimhautdiphtherie vorkommen und in ihrer Lokalisation den Schleimhäuten eng benachbart sind, sondern um primäre, durch Inokulation der Bazillen in die Haut verursachte Hautdiphtherie, ist nach der geringen $\mathrm{Zahl}$ der bisher bekannt gewordenen Fälle diese Ansicht berechtigt.

In den letzten $1 \frac{1}{2}$ Jahren kamen in der Breslauer Universitäts-Hautklinik 5 Fälle von Hautulzerationen mit Nachweis von Diphtheriebazillen zur Beobachtung. Bei 3 derselben wurden diese in Reinkultur gezüchtet und deren Virulenz durch den Tierversuch nachgewiesen.

Der Nachweis der Diphtheriebazillen geschah in der Weise, dab bei der Aufnahme des Patienten von dem unter Vermeidung von Antisepticis gereinigten Ulcus Sekret mit einem sterilen Wattestäbchen abgestrichen wurde und dieses der bakteriologischen Untersuchungsstation des hygienischen Insti- 
tutes der Universität (Geheimrat Flügge) übersandt wurde. Die Kenntnis der dort iublichen Untersuchungsmethode verdanke ich einer persönlichen Mitteilung des Herrn Professor Reichenbach: Das Material wird auf einer Löfflerserumplatte ausgestrichen. Nach 6--8 Stunden wird ein Klatschpräparat hergestellt. Finden sich nach Form und Lagerung typische Diphtheriebazillen, welche die $\mathrm{N}$ e is sersche Doppelfärbung geben und wird dieser Befund nach ca. 24 Stunden unter Berücksichtigung des Aussehens der Kultur gesichert, so erfolgt von dort die Mitteilung des positiven Befundes. Nur in den drei zuletzt beobachteten Fällen wurden auf meine besondere Bitte in dankenswerter Weise die Diphtheriebazillen in Reinkultur gezüchtet und ihre Virulenz durch den Tierversuch nachgewiesen.

Ich werde zunächst über die drei in den letzten Monaten beobachteten Fälle berichten, die ein klinisch einander sehr ähnliches Krankheitsbild, das der echten Hautdiphtherie, boten. Diese Fälle wurden außerdem durch Züchtung der Diphtheriebazillen in Reinkultur und Nachweis ihrer Virulenz durch den Tierversuch ätiologisch sichergestellt.

Fall I. (cf. Abbildung 1.) J., Alfred. Aufnahme am 14./IX. 1906.

Anamnese. War bisher gesund. Von Diphtherie in der Umgebung ist nichts bekannt. Die Erhebung einer eingehend darauf gerichteten Nachforschung war aus äußeren Gründen leider nicht möglich. Die Hautaffektion begann nach Angabe der Mutter vor etwa 4 Wochen mit Rötung an den Stellen, wo jetzt die Geschwüre vorhanden sind. Später wandelten sich diese Stellen in Geschwüre um mit Belägen, die wie Pilzbelag aussahen. Darauf wurde die ganze Fläche wund. Die Geschwüre wurden bereits mit Salben erfolglos behandelt.

Status: Die inneren Organe des $21 / \mathrm{s}$ Jahre alten, leidlich gut genährten Kindes sind gesund. Die Mundschleimbaut ist intakt. An der rechten unteren Bauchseite, parallel dem Ligamentum poupartii, ist ein $3 \mathrm{~cm}$ langes, $1 \frac{1}{2} \mathrm{~cm}$ breites Uleus, begrenzt von leicht wallartig erhabenen, geröteten, nach dem vertieften Geschwürsgrunde zu etwas steil abfallenden Rändern. Diese verlaufen teils polyzyklisch, teils strahlen sie in länglichen schmalen Fortsätzen in die Umgebung aus, sind also wohl zum Teil aus konfluierenden Einzeleffloreszenzen entstanden. Der Geschwürsgrund ist mit einem fest anhaftenden grauweißen bis grangelblichen Belag bedeckt. Ein zweites gleichartiges Ulcus erstreckt sich von der Gegend der Spina anterior superior über die Inguinalgegend, nimmt einen Teil des Skrotums ein und reicht bis $1 \frac{1}{2}$ cm vor die Peniswurzel. Die Umgebung dieses Uleus ist in weitem Umkreise gerötet. Zwisehen 
den beiden großen Geschwüren liegen 2 kleine erbsengroße Geschwüre, die ebenfalls gerötete Ränder und diphtherischen Belag aufweisen. Aus dem oberen Geschwüre wird ein Abstrich entnommen und dem hygienischen Institute übersandt zwecks Untersuchung auf Diphtheriebazillen. Es wuchsen auf $\mathrm{L}$ öfflerserumagar neben zahlreichen Stapbylokokken in geringerer Anzahl Diptheriebazillen. Durch die Verdünnungsmethode gelang es, dieselben in Reinkultur zu züchten.

19./IX. Von einer 24stündigen Bouillonkultur werden sodann $0.5 \%$ des Körpergewichtes einem Meerschweinchen von $200 \mathrm{~g}$ Gewicht am Nacken subkutan injiziert. Tod des Tieres nach 24 Stunden. Sektion: Starkes Ödem und Getäßinjektion unter der Haut des Nackens und Rückens. Pleuritis angedeutet. Starke Hyperämie, dunkelrote Färbung beider Nebennieren, Injektion der oberen Dünndarmpartien.

Am 18./IX. versuchte ich mit Hilfe der von Uffenhejmer kürzlich angegebenen Methode das Diphtherietoxin im Blutserum des kranken Kindes nachzuweisen. Es wurden ca. $3 \mathrm{ccm}$ Blut entnommen, das abgesetzte Serum wurde zentrifugiert und $0 \cdot 3$ Serum zugesetzt zu $3 \cdot 0$ physiologischer Kochsalzlösung, einem Meerschweinchen unter die Bauchhant injiziert. Die Sektion nach Tötung des Tieres 48 Stunden später ergab ein völlig negatives Ergebnis.

Am 18./IX. wurde eines der kleinen Ulzera excidiert und histologisch untersucht.

Mikroskopischer Befund: Leider erwies sich das excidierte Geschwür als eines, das schon deutlich auf dem Wege der Heilung war, dessen ziemlich steil abfallende Ränder. daher auch schon stark epithelisiert waren. Der Grund des Geschwüres, das bis in die Mitte der Cutis hinabreicht, zeigt eine. reichliche zellige Infiltration, aber nur noch geringe Beläge, jedenfalls keine fest anhaftenden pseudomembranösen Auflagerungen, die augenscheinlich durch die voraufgegangene Behandlung (Wasserstoffsuperoxyd) entfernt waren. In der Tiefe des Gewebes wurden Diphtheriebazillen nicht gefunden. Die Beweiskette wurde insofern nicht geschlossen, als der histologische Nachweis der ätiologischen Bedeutung der Bazillen fehlt. Da es sich aber zweifellos um ein heilendes Geschwür handelt, so ist das nicht sehr auffallend.

Verlauf: Das Allgemeinbefinden des Kindes ist anscheinend kaum gestört. Die Abendtemperaturen halten sich in den Tagen vom 14. bis 22./IX. in der Höhe von $36^{\circ} 5$ bis $37.7^{\circ}$, während die Morgentemperaturen vom 19./IX. andauernd unter $37^{\circ}$ bleiben. Die lokale Behandlung bestand in Wasserstoffsuperoxydumschlägen. Nach Sicherung der Diagnose Diphtherie werden am 18./IX. trotz Mangels erheblicher Krankheitserscheinungen 1000. I. E. Diphtherieheilserum iniziert. Eine auffallende Wirkung der Injektion auf die Heilung der Ulzerationen ist nicht zu konstatieren. Dieselben heilen unter den genannten feuchten Verbänden. vom 19./IX. ab unter Verbänden mit Protargolvaseline bald ab. Am 8./X. ist die Heilung unter flacher Narbenbildung vollendet.

F all II. M. Hildegard. Aufgenommen am 24./IX. 1906. 
Anamnese: Eine genaue Anamnese ist nicht zu erheben. Die Infektionsquelle ließ sich nicht nachweisen. Es wurde nur mitgeteilt, dab das Wundsein an den Genitalien seit 6 Tagen bestehe.

Status: Die inneren Organe des $1 \frac{1}{4}$ Jahr alten Kindes sind nicht nachweislich erkrankt. Mundschleimhaut und Rachen nicht. Temperatur $378^{\circ}$. Die rechte große Schamlippe ist geschwollen, fühlt sich etwas derb an und ist dunkelblaurot verfärbt. Auf der Innenseite befinden sich flache, von ausgebuchteten zackigen Rändern begrenzte Ulzerationen mit schmierigen, graugelblichen, fest anhaftendem Belage. Die linke Schamlippe zeigt in etwas geringerem Grade die gleichen Veränderungen. Die Haut auf der Außenseite der rechten Schamlippe trägt eine von einem sehr fest haftenden Belage bedeckte, etwa $4 \mathrm{~cm}$ lange, $1 / 2 \mathrm{~cm}$ breite, strichförmige Ulzeration, deren Ränder infiltriert sind, einen schmalen entzündlichen Hof haben und nach dem tiefen Geschwür zu ziemlich steil abfallen. Die Innenseite des Oberschenkels weist eine ähnliche Ulzeration von etwa Zehnpfennigstückgröße auf.

Im Ausstrichpräparat werden keine Gonokokken gefunden, weder im Geschwür, noch in Urethra und Vulva. Ein Abstrich aus dem Uleus an der Innenfläche des Oberschenkels wird dem hygienischen Institut zur Untersuchung übersandt. Daselbst werden am 25./IX. auf dem in der vorigen Krankengeschichte eingehend geschilderten Wege Diphtheriebazillen nachgewiesen, deren Virulenz durch einen Tierversuch in ganz analoger Weise erwiesen wurde: das von 220 auf $170 \mathrm{~g}$ abgemagerte Meerschweinchen ging 22 Stunden nach der Injektion zu Grunde. Die Sektion ergab den oben geschilderten typischen Befund.

Verlauf: Das Allgemeinbefinden des Kindes ist kaum beeinträchtigt. Lokal werden Wasserstoffsuperoxydumschläge appliziert.

25./IX. Temperatur Früh $36^{\circ} 0^{\circ}$, Abends $36^{\circ} 7^{0}$.

26./IX. Temperatur Früh $36.8^{\circ}$, Abends $37 \cdot 3^{\circ}$. Injektion von 1500

I. E. Diphtherieserum. Die Temperatur geht in den nächsten Tagen nicht über $37 \cdot 3^{0}$, nur am 30./IX. und 1./X. noch eine Steigerung auf $37 \cdot 6$ und $37 \cdot 9^{\circ}$, dann wieder Abfall.

2./X. Die Geschwüre sind gereinigt. Lokal: Protargolvaseline 5\%. 11./X. Sämiliche Ulzera sind geheilt, Entlassung.

Fall III. B., Gertrud. Aufgenommen am 28./XI. 1906.

A namnese: Das $2 \frac{1}{2}$ Jahre alte Kind war seit März d. J. wiederholt in Behandlung der chirurgischen Universitätspoliklinik. Von dort wurden uns folgende Angaben übermittelt: 9. März 1906: Tbc. maxillae sin., verschiedentlich punktiert. AbszeB am Processus zygomaticus. Am 28. Aug. Punktion eines Abszesses in der Parotisgegend, Injektion von Jodglyzerin. Am 17. Okt. Tbe. Abszeß am linken unteren Orbitalrand punktiert (Eiter) und Jodoformglyzerin injiziert.

Am 28/XI. wurde das Kind wegen multipler Hautulzeration der Hantklinik überwiesen. Nach Angabe der Mutter sollen sich aus den "Beulen" auf der linken Wange, deren Narben zu sehen sind, wiederholt im Laufe des Sommers Geschwüre gebildet haben, die langsam zuheilten. 
Das Geschwür an der linken Stirnseite soll etwa 2 Wochen bestehen und nicht ganz solange die Geschwüre in der Genitocraralgegend. Von einer Gelegenheit, bei der das Kind mit Diphtheriekranken in Berührung gekommen sein könnte, ist der Mutter nichts bekannt.

Status: Das Kind ist für sein Alter ganz gut entwickelt. Das Allgemeinbefinden ist kaum beeinträchtigt. Die Temperatur ist nur sehr wenig erhöht $\left(37 \cdot 5^{0}\right)$. Die Schleimhäute der Mundhöhle und Genitalien sind ohne Veränderungen.

Auf der linken Wange sieht man 3 fingernagelgroße, leicht bläulich verfärbte, etwas eingezogene Narben. Im Bereich von einer dieser Narben in der linken Schläfengegend ist ein fast pfennigstückgroßes Ulcus von unregelmäßiger Form, nach 2 Seiten hin in kleinen zungenförmigen Ausläufern sich fortsetzend. Ein festanhaftender grauer Belag füllt es fast aus: später nach Loslösung desselben sieht das Ulcus tief, wie mit dem Locheisen ausgeschlagen aus. Die Umgebung ist nur wenig gerötet, kaum infiltriert.

Auf der linken großen Schamlippe sind 2 ca. $1 \frac{1 / 2}{c m}$ lange und $1 / 4 \mathrm{~cm}$ breite parallel der Längsrichtung der Schamlippen verlaufende und nach außen davon 2 kaum stecknadelkopfgroße Ulzera. Die rechte Schamlippe trägt ein linsengroßes, sehr unregelmäßig geformtes und 4-5 kleinere gleichartige Ulzera. An der Außenseite der im ganzen etwas geschwollenen rechten Schamlippe ist ein $21 / 2 \mathrm{~cm}$ langes, $3 / 4 \mathrm{~cm}$ breites Ulcus, dicht dabei, schon am Oberschenkel, ein pfennigstückgroßes Ulcus.

Ein größeres (etwa talergroßes), sehr unregelmäßig geformtes Ulcus nimmt die rechte lnguinalbeuge ein. Unterhalb desselben befinden sich mehrere kleinere und kleinste Geschwüre.

Die Umgebung der Geschwüre ist in weitem Umkreise gerötet, die Haut intertriginös verändert. Nirgends sind Rhagaden, Knötcheln oder Pusteln zu erkennen. Die Geschwürsränder sind kaum erhaben. Der Geschwürsgrund ist grau bis graugelblich, hier und da noch mit einem festanhaftenden Belage versehen.

An der Ulnarseite des linken Unterarmes, an der Grenze zwischen mittleren und oberen Drittel ist eine wallnuligroße, ziemlich scharf abgegrenzte derbe Anschwellung mit 2 kleinen Öffnungen, aus denen sich sanguinolentes, dünnfiüssiges Sekret entleert. Die Haut darüber ist leicht gerötet.

Bakteriologischer Befund: Es werden von 3 verschiedenen Stellen Sekretabstriche dem hygienisehen Institut zwecks Untersuchung auf Diphtheriebazillen übersandt:

1. Von dem Ulcus an der Stirn. Es werden vorwiegend Kokken, daneben in großer Menge typische Diphtheriebazillen gefunden, die in Reinkultur gezüchtet werden.

Von dieser mir zur Verfügung gestellten Reinkultur legte ich eine 24stündige Bouillonkultur an und injizierte $1.0 \mathrm{bzw}$. 1.25 davon zwei Meerschweinchen von 200 bzw. $250 \mathrm{~g}$ Körpergewicht. 
Die Tiere zeigten nach 48 Stunden keinerlei Veränderungen des Allgemeinbefindens; sie wurden zu dieser Zeit getötet. Der Sektionsbefund war negativ.

An 6./XII. injizierte ich nochmals $0.9 \mathrm{~cm}$ einer 24stündigen Bouillonkultur unter die Bauchhaut eines Meerschweinchens von $175 g$ Gewicht. Nach 4 Tagen starb das Tier. Sektionsbefund: Starkes hämorrhagisches Ödem der Bauchhaut an der Injektionsstelle. Dunkelrote Verfärbung der linken Nebenniere, deutliche Hyperämie der rechten Nebenniere. Injektion der Gefäße in den oberen Dünndarmpartien.

Ein zweites, $250 \mathrm{~g}$ schweres Meerschweinchen, daß am gleichen Tage mit 1.25 cem der 24stündigen Bouillonkultur injiziert wurde, starb nach 3 Tagen. Sektionsbefund: Starkes hämorrhagisches Ödem der Bauchhaut an der Injektionsstelle. Tiefdunkelrote Verfärbung beider Nebenvieren. Injektion der oberen Dünndarmpartien.

2. Von dem Abstrich aus einem größeren Ulcus in der Inguinalbeuge wuchsen von vornherein vorwiegend Diphtheriebazillen. (Nahezu Reinkultur.) Die Reinkultur wurde mir vom hygienischen Institut zur Verfügung gestellt. $1 \mathrm{ccm}$ einer 24stündigen Bouillonkultur wurden einem $200 \mathrm{~g}$ schweren Meerschweinchen injiziert. Das Tier starb nach $4^{1 / \mathrm{s}}$ Tagen. Sektionsbefund: Ausgesprochenes hämorrhagisches Ödem der Bauchhaut an der Injektionsstelle. Mäßige Hyperämie beider Nebennieren.

Das Sekret aus der Fistel am linken Unterarme wurde ebenfalls auf Diphtheriebazillen im bygienischen Institut untersucht. Der Befund war negativ. Auch Tuberkelbazillen ließen sich nicht nachweisen.

Verlauf: Die Abendtemperaturen sind in den ersten Tagen ein wenig erhöht (am ersten Tage auf $379^{\circ}$, am 2.-4. Tage auf $374^{0}$, von da an bleiben sie dauernd unter $37^{\circ}$ \%. Nach Sicherung der Diagnose Hautdiphtherie durch Diphtheriebazillenbefund werden am 1./XII. 1000 I. E. Behringsches Diphtherieserum injiziert. Ein Einfluf auf das Allgemeinbefinden und die Temperatur ließ sich unter den an sich günstigen Verhältnissen natürlich nicht konstatieren. Die Hautulzerationen heilten allerdings ziemlich bald (in etwa 12 Tagen) ab, sie wurden aber auch intensiv antibakteriell behandelt (anfangs $\mathrm{H}_{2} \mathrm{O}_{2}$-Umschläge, später Verbände mit $10 \%$ iger Protargolvaseline).

Diese 3 Fälle repräsentieren das typische Bild der Hautdiptherie, das uns nach früheren Erfahrungen schon makroskopisch die Diagnose Diphtherie nahelegte.

Dem Infektionsmodus nach ist wohl jede "primäre Hautdiphtherie" eine Inokulationsdiphtherie, die auf dem Boden von intertriginösen Ekzemen, Rhagaden, Kratzeffekten und anderen kleinen Hautläsionen entsteht. Die Entstehungsweise ist also eine ähuliche, wie sie für das sogen. Ekthyma angenommen wird und es wahrscheinlich auch ist. (Neis ser.) Bevorzugt werden solche Gegenden der Haut, die der Mazeration ganz 
besonders ausgesetzt sind und zu jeder Art von Wundsein neigen, wie die Genitocrural- und -Analregion, ganz besonders bei Kindern, deren Haut gegen alle die genannten Schädigungen noch bedeutend empfindlicher ist als die der Erwachsenen.

In diesen 3 Fällen ließ sich nichts Bestimmtes über die Infektionsquelle ermitteln. Die Lokalisation in der Umgebung der Genitalien, an der unteren Bauchgegend, in der Inguianalbeuge, an der Innenfläche der Obnrschenkel und den großen Labien läßt jedoch auch hier die Annahme als wahrscheinlich erscheinen, daß es sich um die erwähnte Entstehungsweise handelt, da eben gerade diese Gegenden bevorzugte Sitze intertriginöser Ekzeme sind. Das gleichzeitige Bestehen einer Schleimhautdiphtherie kann rollständig fehlen, wie diese Fälle lehren. Die rorhandenen Effloreszenzen sind ausschließlich Ulzera, anfangs ronkleinsten Dimensionenund außerordentlich oberflächlich. Bei längerem Bestande konfluieren sie und werden meistungewöhnlich groß. Ihre Form ist sehr unregelmä $\beta i g$, da die Ränder teils im polyzyklischen Bogen, teils in tiefen schmalen Buchten in das Gebiet der gesunden Haut hineinverlaufen. Die Ränder sind leicht infjltriert, intensiv gerötet und nur hier und da etwas unterminiert, meist fallen sie zu dem vertieften Geschw ürgrund hinziemlich steil ab. Der Geschwürsgrund ist mit einem grauweißen, fest anhaftenden diphtherischen Belage bedeckt, der allein den Gedanken ohne weiteres nahe legt, die Diagnose Diphtherie der Haut in Betrachtzuziehen. Fötor ist, wenn überhaupt vorhanden, nur gering. Nach Reinigung der Ulzera kommt es schnell zu kräftiger Granulationsbildung; die Heilung geht relativ schnell von statten. Die Reaktion der Ulzerationen auf die Diphtherieseruminjektionen läßt sich an so wenigen Fällen schlecht beurteilen, da es möglich ist, daß die Heilung unter der gleichen Therapie auch ohne Seruminjektion ebenso schnell ron statten gegangen wäre. Ebensowenig ließ sich ein Einfluß der Diphtherieseruminjektionen auf den Temperaturverlauf konstatieren. Das er- 
scheint nicht verwunderlich, da sich die Temperatur in allen Fällen nie über eine abendliche Steigerung von $37 \cdot 9^{\circ}$ erhob. Die Kinder zeigten keineswegs eine schwere toxische Beeinflussung des Gesamtorganismus. Die Allgemeinerscheinungen waren also nicht, wie z. B. Baginsky angibt, ganz diejenigen der Diphtherie, sondern bei beiden Kindern war das Befinden nur sehr wenig beeinträchtigt. Es ist deshalb auch nicht wunderbar, daß ein Nachweis des Toxins im Blute nicht gelungen ist.

Bietet somit das Krankheitsbild durch Lokalisation, Form und Begrenzung, sowie besonders durch den Belag der Geschwüre einige charakteristische Merkmale, so ist dennoch eine sichere Diagnose ohne Nachweis virulenter Diphtheriebazillen nicht möglich. Vor Besprechung der Differentialdiagnose möchte ich einen 4. Fall erwähnen, der unter dem klinischen Bilde des Ekthyma infantile verlief:

Fall IV. (cf. Abbildung 2, Moulage Nr. 763). Cz., Alfred, 3 Jahre alt, Aufnahme am 22./V. 1905.

Anamnese: In der Familie ist Tuberkulose wiederholt vorgekommen. Vor einem Jahre hatte das Kind Masern, vor einem halben Jahre Keuchhusten. Vor $1 \frac{1}{2}$ Monaten wurde es laut Mitteilung der Universitätskinderklinik daselbst einige Male wegen „Phlyktaenen, Bronchitis und Otitis media (exsudative Diathese)" poliklinisch behandelt. Seit 6 Wochen besteht die Hauterkrankung. Zuerst bildeten sich nach Angabe der Mutter Blattern und Krusten auf dem Kopfe, dann wurden Arme und Beine befallen, zum Schluß der Bauch, wo sich wasserbelle Blasen bildeten. Fieber soll in der ganzen Zeit bestanden haben, die Gewichtsabnahme in den letzten 2 Wochen besonders groß gewesen sein. Der allgemeine Befund der Universitätskinderklinik ist folgender : „Die Augen sind zur Zeit frei, die genauere Lungenuntersuchung ist des Verbandes wegen nicht möglich, eine Bronchitis besteht jedenfalls noch immer. Außerdem sind die Rachenorgane stark gerötet, die Ohren sind zur Zeit auch nicht der Untersuchung zugänglich. Die Milz ist vergrößert. Die Temperaturerhöhung ist wohl von seiten der Affektion der Luftwege zu erklären, vielleicht im Verein mit den ausgedehnten Hautulzerationen." (Freund.)

Temperatur Früh $37 \cdot 5^{0}$, Abends $38^{\circ} 2^{\circ}$. Auf der Haut des oberen Drittels des rechten Oberschenkels beiderseits am Gesäß, hinter dem Ohre, an der Außenfläche des linken Unterschenkels, an der Ulnarseite des rechten Unterarmes mehrere bis pfenniggroße, teils mit Granulationen, teils mit nekrotischen Fetzen bedeckte, scharf ausgeschnittene, zum Teil die ganze Oberhaut 
durchsetzende rundliche Geschwüre, die sich gegen die gesunde Umgebung mit einem schmalen, ziemlich lebhaft geröteten, leicht erhabenen Rande abgrenzen. Ein über pfenniggroßes, längliches, tiefes Ulcus mit unregelmäBigem Grunde, der einen leichten graugeblichen Belag trägt, mit zum Teil überbängenden Rändern, befindet sich im Oberschenkel in der rechten Genitocruralfalte.

23./V. Es wird von dem letzterwähnten Ulcus ein Abstrich gemacht und dem hygienischen Institut übersandt. Am 24./V. erfolgte die Antwort, $\mathrm{da} B$ in dem übersandten Material echte Diphtheriebazillen nachgewiesen wurden, außerdem in der Mehrzahl Staphylokokken. Daraufhin werden 1000 I. E. Diphtherieserum injiziert. Am 27./V. wird ein zweiter Abstrich aus dem mit Borsäure feucht verbunden gewesenen Ulcus dem hygienischen Institut übersandt. Dieses Mal wurden nur Staphylokokken konstatiert. Die etwas gereinigte Ulzera werden jetzt mit Protargolvaseline verbunden.

Am 29./V. tritt leichte Benommenheit ein. Am 30./V. wird die Benommenheit stärker. Befund der Univ.-Ohrenklinik: „Rechtsseitige alte perforative Otitis media mit akutor Exacerbation." Operation wird vorgeschlagen aber von der Mutter verweigert. Befund der Univ.-Augenklinik: „Neuritis optica dextra. Abducensparese links. Verdacht auf Neuritis optica links." Am gleichen Tage tritt tiefes Koma ein, klonische Krämpfe der Gesichtsmuskulatur, zeitweise auch aller Extremitäten. Exitus. Die Temperatur schwankte während dieser Zeit zwischen $37^{\circ}$ Früh und 37.8 bis $38^{\circ} 8^{\circ}$ Abends.

Pathologisch-anatomische Diagnose (Path. Inst. der Universität): Meningitis, Otitis media chronica dextra perforata. Neuritis optica dextra. Meningitis tbe. Tbc. pulmonum, Peritonitis caseosa, Tbc. miliaris splenis, hepatis, peritonei, diaphragmatis, ulcera tbc. ilei. Hautulzera cf. status.

Das klinische Bild dieses Falles war das des Ekthyma infantile. Auffallend ist die Lokalisation der größeren Geschwüre in der unteren Bauch- und Inguinalgegend, während für gewöhnlich beim Ekthyma die Streckseiten der Extremitäten und der Rücken ganz besonders befallen zu sein pflegen. Die Moulage läßt die Primäreffloreszenzen deutlich erkennen. Es sind kleine Pusteln mit entzündlichem Hofe. Bei weiterem Wachstum büßen sie die Blasendecke ein und werden zu meist flachen Ulzerationen. Die Ulzera sind rund, die Ränder sind mit einem schmalen geröteten Hof umgeben. Der Grund der Geschwüre ist mit einem gelblichen Belag bedeckt; stellenweise liegen frische Granulationen zu Tage. Nnr in dem Belag einer der größeren Ulzerationen wurden Diphtheriebazillen durch Färbung und Kultur nachgewiesen. Da Züchtung in Reinkultur und Nach- 
weis der Tierpathogenität nicht ausgeführt wurden, die Diphtheriebazillen außerdem nur in einem einzigen Ulcus nachgewiesen wurden, so kann ich diesem Befunde in Bezug auf die Ätiologie der gesamten Hautaffektion keine große Bedeutung beimessen. Es bleibt natürlich auch der Einwand, daß es sich in diesem Falle nicht um virulente Diphtheriebazillen gehandelt hat und daß möglicherweise ihre Ansiedlung als eine rein sekundäre $\mathrm{zu}$ betrachten ist.

Kehren wir von diesem ekthymaähnlichen Falle zurück zur Besprechung der Differentialdiagnose der typischen echten Hautdiphtherie, wie sie uns in den 3 ersten F'ällen entgegentrat, so haben wir folgende Erkrankungen in Betracht zu ziehen.

Nosocomialgangrän (Hospitalbrand): Diese Erkrankung führte bekanntlich früher den Namen Hautdiphtherie. Von dem Begriff der echten, durch Löfflersche Diphtheriebazillen bedingten Hautdiphtherie wurde sie schon früh durch das Urteil namhafter Chirurgen losgelöst. Tavel erinnert an 2 Fälle, die Kocher als typische Wunddiphtherie ansprach und die sich bakteriologisch nicht als solche erweisen ließen. Matzenauer sagt über das Aussehen dieser Geschwüre der Nosocomialgangrän: „Die Geschwüre charakterisieren sich insbesondere durch die Auflagerung eines schmutzig graugrünlichen bis schwärzlichen Belages von variabler, manchmal mehrerer Zentimeter Dicke. Der Belag ist gelatinös, pastös, schlammartig oder zunderartig zerfallen, siebt aus wie ein Schorf mit ätzender Säure. Rund um den Geschwürsgrund ist ein schmaler hellroter Entzündungshof; die Geschwüre verbreiten einen penetrant fauligen Geruch. Dabei bestehen zumeist Fiebererscheinungen.

Diese Geschwüre können am Genitale sowohl selbständig und primär als solche auftreten oder sich zu einer bereits bestehenden venerischen Affektion hinzugesellen, wobei zugleich diese letztere ibren Charakter verliert und die Gangrän substituiert wird."

Diese Gangränformen treten nach den von ihm geschilderten Fällen nur beim Erwachsenen auf und bieten in der

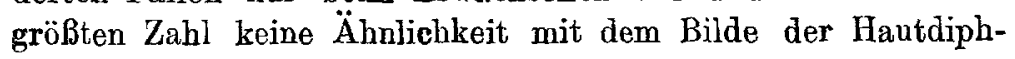


therie, d. h. sie lassen den fest anhaftenden, grauweiß- bis graugelblichen diphtherischen Belag vermissen, und das Bild der Gangrän mit ihrem penetrant fauligen Geruch tritt in den Vordergrund. Róna hat in derartigen Fällen vergeblich versucht den Diphtheriebazillus zu züchten. Da aber die Geschwüre der Nosocomialgangrän auch das Bild der echten diphtherischen Hautgeschwüre annehmen können, wie aus den Beschreibungen der älteren Autoren hervorgeht, so ist gerade bei der Lokalisation solcher Ulzerationen in der Genitocrural- und -analregion, an der unteren Bauchseite und den Oberschenkeln, ganz besonders, wenn die Erkrankung Kinder betrifft, die Möglichkeit einer Infektion mit echten Diphtheriebazillen in Betracht zu ziehen.

Bei der Hautdiphtherie ist bemerkenswert die eigenartige Form der Geschwïre, wie sie alle 3 Fälle zeigen. (cf. Abbildung 1.) Diese im ganzen höchst unregelmäßige Gestalt mit den bogenförmigen Ausbuchtungen, hier und da in der Form länglicher Streifen in die gesunde Haut sich fortsetzend, vermißt man bei der Nosocomialgangrän.

Die Abgrenzung ron Ekthyma infantile ergibt ein Vergleich der beiden abgebildeten Fälle. Bei Ekthyma sind die Primäreffloreszenzen als Pusteln deutlich erkennbar. Die Effloreszenzen sind zahlreich und zeigen die rerschiedenen Entwicklungsphasen der im ganzen flachen Geschwüre, welche zumeist kreisförmig begrenzt sind. Bei der Diphtherie sehen wir einzelne große tiefe Ulzerationen mit anfangs diphtherischem Belag und die wenigen winzigen Ulzera zeigen genau die Charaktere der großen. Der ProzeB scheint von vornherein als ganz flache Ulzeration mit kaum erhabenem oder infiltriertem Rand in geröteter (intertriginöser) Umgebung zu entstehen und abgesehen ron Tiefe und Ausdehnung genau die gleichen Charakterezu besitzen, wie späterhin.

Von Jod- und Bromtoxikodermien, die zur Bildung schlaffer großer Blasen führen können, nach deren Zerfall verschieden große runde, flache oder auch tiefere Ulzerationen entstehen, sind die echten diphtherischen Hautulzera wohl leicht abzugrenzen. 
Mit dem Ulcus molle kann die Form der Ulzera gewisse Ähnlichkeiten bieten. Beim Ulcus molle haben wir den kraterförmigen Substanzverlust, wie mit dem Locheisen ausgeschlagen. Die Ränder sind bei längerem Bestande etwas unterminiert. Diese und der Geschwürsgrund sind etwas speckig belegt, entzündlich gerötet und geschwellt. Die diphtherischen Geschwüre sind am Rande erheblich stärker infiltriert. Die Geschwüre erscheinen tiefer und außerdem ist in einem gewissen Stadium stets der graue, fest anhaftende Belag vorhanden. Die Geschwüre nehmen meist größere Dimensionen als das Ulcus molle an und sind nicht so multipel wie dieses. Auch fehlt eine stärkere Beteiligung der regionären Drüsen.

Eine Ähnlichkeit mit ulzerierten syphilitischen Papeln war nicht vorhanden. Papelähnliche Effloreszenzen fehlten völlig.

Das zwischen Syphilis und Diphtherie als Ursache von Hautulzerationen gelegentlich ein enger Zusammenhang bestehen kann, dafür scheint ein 5. von uns beobachteter Fall zu sprechen: In einem phagedänischen Uleus des Penis wurden zuerst Diphtheriẹbazillen und einige Zeit später Spir. pallidae nachgewiesen. Da hier Reinkultur von Diphtheriebazillen nicht gezüchtet wurde und auch der Tierversuch fehlt, so möchte ich auch diesem Fall nicht die gleiche Bedeutung wie den drei ersten zumessen.

Fall V. R., Hermann, 31 Jahre alt. Aufgenommen 27./V. 1905.

Anamnese: Früher gesund. Vor 8 Wochen bemerkte er eine schwarze Blatter auf dem Dorsum penis, die trotz rationeller Behandlung sich zu einem großen Geschwür entwickelte. Seit 3 Wochen leidet er an Schlaflosigkeit, Appetitlosigkeit und Schwächegefühl. Er bekam in den letzten Wochen von seinem Arzt 6 Hg-Injektionen.

Status: Aussehen blaß, abgemagert. Das Dorsum penis und ein 1/2 handtellergroßer Bezirk des Mons veneris sind von einem verwahrlosten, mit Haaren verklebten, sehr übel riechenden, tiefen Uleus eingenommen. Dasselbe ist durch relativ schmale, gerötete und infiltrierte, erhabene, steil abfallende Ränder umgrenzt. Die Geschwürsfäche ist von einem schmierigen erweichten grauen Belag bedeckt. Die Inguinal-, Zervikal- und Cubitaldrüsen sind geschwollen, dabei indolent. Auf der Schleimhaut der Unterlippe eine plaqueähnliche Erosion. In einem dem hygienischen Institute übersandten Abstrich werden Diphtheriebazillen nachgewiesen (keine Reinkultur, kein Tierversuch). Wiederholte Untersuchung auf Spirochaeten ergaben stets ein negatives Ergebnis.

Die Begrenzung und Reinigung des Geschwüres geht sehr langsam von statten. Protahierte Sitzbäder mit Kal. perm.; Jodoform- und Schwarzsalbeverbände. Gegen Ende Juli nach fast 3monatlicher Behand- 
lnng hat das Ulcus einen etwas veränderten Charakter angenommen. Es ist im ganzen sehr verkleinert, der Grund ist gereinigt, die Ränder sind fiacher und haben einen mehr bräunlichen Farbenton bekommen. Am 23./VII. gelingt es im Abstrich aus den Randpartien des Ulcus Spir. pallidae nachzuweisen. Gleichzeitig treten am Skrotum syphilitische Papeln auf, so dab die Diagnose Syphilis nunmehr gesichert ist. Unter energischer Hg-Behandlung erfolgt jetzt schnelle Heilung.

15./VIII. geheilt entlassen.

Das Aussehen des Ulcus glich im Anfang den Geschwüren der Nosocomialgangrän, wie sie von Matzenauer und Róna als besonders in der Genitoanalregion vorkommend beschrieben werden. Von den Geschwüren in den ersten beiden Fällen unterschied es sich wesentlich durch den weichen, milfarbigen, einen üblen Fötor verbreitenden Belag, des Vorherrschen des gangränösen phagedänischen Charakters. Da dieser Fall mindestens die Wahrscheinlichkeit der ätiologischen Bedeutung der gefundenen Diphtheriebazillen nahe legt, oder wenigstens darauf hinweist, daß der eigentümliche Verlauf der Ulzeration mit durch die Wucherung der Diphtheriebazillen bedingt war, so sollte in ähnlichen Fällen außer nach den von Matzenauer und $R$ ón a beschriebenen Bazillen und fusiformen Bazillen auch stets kulturell nach Diphtheriebazillen geforscht werden. Die Wichtigkeit des Nachweises etwa vorhandener Diphtheriebazillen liegt auf der Hand. Natürlich muß deren Nachweis in Reinkultur und ihre Virulenzprüfung durch den Tierversuch gefordert werden.

Die Zahl der in den letzten Jahren bekannt gewordenen Fälle von Hautdiphtherie mit Nachweis Löffler scher Diphtheriebazillen ist gering. Üher die früheren Fälle bis 1900 ist von Leick kurz berichtet. Zu der Kategorie unserer Fälle 2 und 3 gehört der erste bakteriologisch als Hautdiphtherie sicher gestellte Fall Ernst $\mathrm{Neissers.} \mathrm{Es} \mathrm{bestanden} \mathrm{neben}$ schwerer Schleimhautdiphtherie in der Ausdehnung von $10 \mathrm{zu} 4 \mathrm{em}$ weißliche pseudomembranöse Beläge an der Rima ani bei einem 5 Jahre alten Knaluen; ferner der Fall Schottmüllers: Aus einer zerkratzten Aknepustel bildete sich ein großes Geschwür. In der Mitte der rechten Inguinalfalte, sowohl ober- wie unterhalb des Ligamentum Poupartii bestand ein je etwa feigengroßer Defekt. Der Grund war mit grauweißlichen mißfarbenen Fetzen bedeckt. Fast im ganzen Verlauf der Schenkelbeuge und Umgebung war die Haut entzündlich gerötet, zum Teil der Epidermis beraubt und mit dünnen, grauweißlichen, membranösen Auflagerungen bedeckt. Es wurden Diphtheriebazillen auch nachgewiesen im gesunden Rachen des Kindes. Das Kind war schwer krank, blaß und elend, Fieber bestand nicht. 
Ebenso gehört dahin der von A. W. K. Müller beschriebene, ein zehnjähriges Mädchen betreffende Fall. Hier bestanden neben Halsdiphtherie, Paronychie und diphtherischen Auflagerung und Ulzerationen der Vulva zu beiden Seiten der Nates an symmetrischen Stellen mehrere etwa linsengroße Substanzverluste, teils mit Krusten bedeckt, teils mit eitrigem, von geröteten Rändern umgebenem Grad. Es wurden Diphtheriebazillen in Reinkultur gezüchtet.

Ferner möchte ich noch erwähnen den Fall Hassensteins, wo ein Säugling an Stelle des Nabels ein kraterförmiges, speckig glänzendes Geschwür hatte. Fieber fehlte. Das Allgemeinbefinden war gut. Die Übertragung hatte nachweisbar durch eine Hebamme stattgefunden.

Endlich zwei von $\mathrm{Schwab}$ publizierte Fälle mit exaktem Diphtheriebazillennachweis und im ganzen subakutem Verlauf, bei denen jedoch die Hantaffektion die großen Labien nicht überschritt.

Eine neuere Arbeit ron Adler über Hautdiphtherie war mir leider nur in einem kurzen Referat zugänglich.

Zur Bildung ähnlich charakterisierter diphtherischer Ulzerationen im Anschluf an Verwundungen kam es in Fällen Brunners und einem Falle von Abel, welche Fingerverletzungen betreffen, Scholtz führt einen Fall aus der Breslauer Hautklinik an, wo es nach Durchstechen eines Ohrläppchens zum Zwecke des Anbringens von Ohrringen zu einem diphtherischen Geschwür mit Defekt des Ohrläppchens kam. Diphtherie der Lidhaut beobachtete eine größere Zahl von Autoren. Hervorheben möchte icb einen Fall Uhthoffs, wo bei bestehender Diphtherie der Conjunctivae auch von der äußeren Haut, ca. $2 \mathrm{~cm}$ vom unteren Lidrand entfernt, von einer Stelle, wo dieselbe sich noch mit leichten Krusten und Borken bedeckt zeigte, Impfmaterial entnommen und in exakter Weise virulente Diphtheriebazillen nachgewiesen wurden. „Es zeigt also dieser Fall jedenfalls, " wie Uhth off sagt, "daß bei Conjunctivitis diphtheritica, wenn gleichzeitig Gesichtsekzem in der Umgebung des Auges besteht, die virulenten Bazillen auch auf der äußeren wunden Haut vegetieren können."

In den sonst beobachteten Affektionen der Haut, wo Diphtheriebazillen nachgewiesen wurden, handelte es sich um Infektionen tiefer Wunden, Abszesse und anderer subkutaner Infektionen. Hier kommt es nicht zur Bildung des typischen diphtherischen Belages, und diese Affektionen unterscheiden sich in keiner Weise von den Bildern, die durch andere Eiter- 
erreger hervorgerufen werden können. Dahin gehören die beiden anderen Fälle Brunners, die Fälle Tavels u. a. m. Bei diesen uncharakteristischen Eiterungsprozessen wird es immer nur Sache des Zufalls sein, daß gelegentlich einmal Diphtheriebazillen nachgewiesen werden, und nur die naheliegende Möglichkeit einer Infektion von einem Diphtherieherd aus wird diese Untersuchung veranlassen.

Das Studium unserer 3 ersten Fälle lehrt, daß es möglich ist, bei einer gewissen Art von Hautulzerationen allein aus dem klinischen Bilde mit großer Wahrscheinlichkeit die Diagnose Hautdiphtherie zu stellen. Die unregelmäßige Form der Geschwüre, der diphtherische Belag, die Lokalisation bieten die Hauptanhaltspunkte dafür. In allen solchen Fällen soll versucht werden, durch Züchtung von Reinkulturen und Virulenzprüfungen die Diphtheriebazillen nachzuweisen. Bei Lokalisation in der Genitocrural- und Analgegend soll auch jedes für Hautdiphtherie nicht ganz typische Ulcus, das z. B. den Charakter des Ekthyma infantile oder den der Nosocomialgangrän trägt, auf Diphtheriebazillen untersucht werden. Nur so wird es möglich sein, frühzeitig in vielen Fällen eine ätiologische Therapie einzuleiten und durch Isolierung des Kranken den Krankheitsherd zu beschränken.

Zum Schluß ist es mir eine angenehme Pflicht, Herrn Oberarzt und Privatdozenten Dr. Zi eler, derzeitigem stellvertretenden Direktor der Klinik, für die Überweisung dieser Arbeit und seine mannigfache Unterstützung bei Abfassung derselben meinen ergebensten Dank auszusprechen.

\section{L i t e ratur.}

1. Neisser, Ernst. Ein Fall von Hautdiphtherie. Dtsch. med. Wochenschr. Nr. 27. p. 703.

2. Brunner, C. Über Wunddiphtherie. Berl. klin. Wochenschr. 1894. Nr. $23-24$.

3. Brunner, C. Eine weitere Beobachtung von Wunddiphtherie Berl. klin. Wochenschr. 1894. Nr. 13.

4. Abel, R. Ein Fall von Wunddiphtherie und Nachweis von Diphtheriebazillen. Dtsch. med. Wochenschr, 1894. Nr. 26. 
5. Schottmüller, H. Ein Fall von Wunddiphtherie etc. Dtsch. med. Wochenschr. 1895. Nr. 17.

6. Seitz J. Diphtheriebazillen in einem Panaritium. Korrespondenzbl. für schweizer Ärzte. 1899. Nr. 29. Zit. nach Ba umgartens Jahresb.

7. Hassenstein, W. Ungewöhnliche Formen diphtherischer Erkrankungen übertragen durch eine Hebamme. Dtsch. med. Wochenschr. 1899. p. 406.

8. Müller, A. W. K. Über seltenere Lokalisationen der Diphtheriebazillen auf Haut und Schleimhaul. Dtsch. med. Wochenschr. 1899. Nr. 6.

9. Leick. Dtsch. med. Wochenschr. 1900. pag. 196.

10. Tavel. Über Wunddiphtherie. Dtsch. Zeitschr. für Chir. Bd. LX. pag. 460 .

11. Steffens, P. Ein Fall von Lidgangrän mit Diphtheriebazillenbefund. Klin. Monatsh. f. Augenheilk. 1900. pag. 399.

12. Uhthoff, W. Ein weiterer Beitrag zur Conjunctivitis diphtheritica. Berl. klin. Wochenschr. 1894. Nr. 34.

13. Schwab, Th. 2 Fälle von ansgedehnten Ulzerationsprozessen an Mund und Genitalien, hervorgerufen durch Diphtheriebazillen. Arch. f. Derm. und Syphilis. Bd. LXVIII. pag. 101.

14. Matzenauer. Zur Kenntnis und Ätiologie des Hospitalbrandes. Arch. f. Derm. und Syphil. :Bd. LV. pag. 67.

Bd. LXXI.

15. Rona, S. Nosocomialgangrän. Arch. f. Derm. und Syphilis.

16. Scholtz, W. Die deutsche Klinik. 1905. Bd. X. II. Teil.

17. Baginsky. Diphtherie und diphther. Croup. Notnagels speziell. Pathol. u. Ther. Bd. I.

18. Caposi. Pathologie und Therapie der Hautkrankheiten. Handb.

19. Neisser, A. Krankheiten der Haut. In Ebstein Schwalbes

20. Adler. Über Hautdiphtherie im Kindesalter. Berl. klinische Wochenschr. 1904. Nr. 20/28 zit. nach Arch. f. Derm, und Syph. Band LXXVI. pag. 132.

21. Uffenheimer. Der Nachweis des Toxins im Blute des Díphtheriekranken. Münch. med. Wochenschr, 1906. Nr. 33.

Die Erklärung der Abbildungen auf Taf. VII ist dem Texte zu entnehmen. 
$\stackrel{乛}{2}$
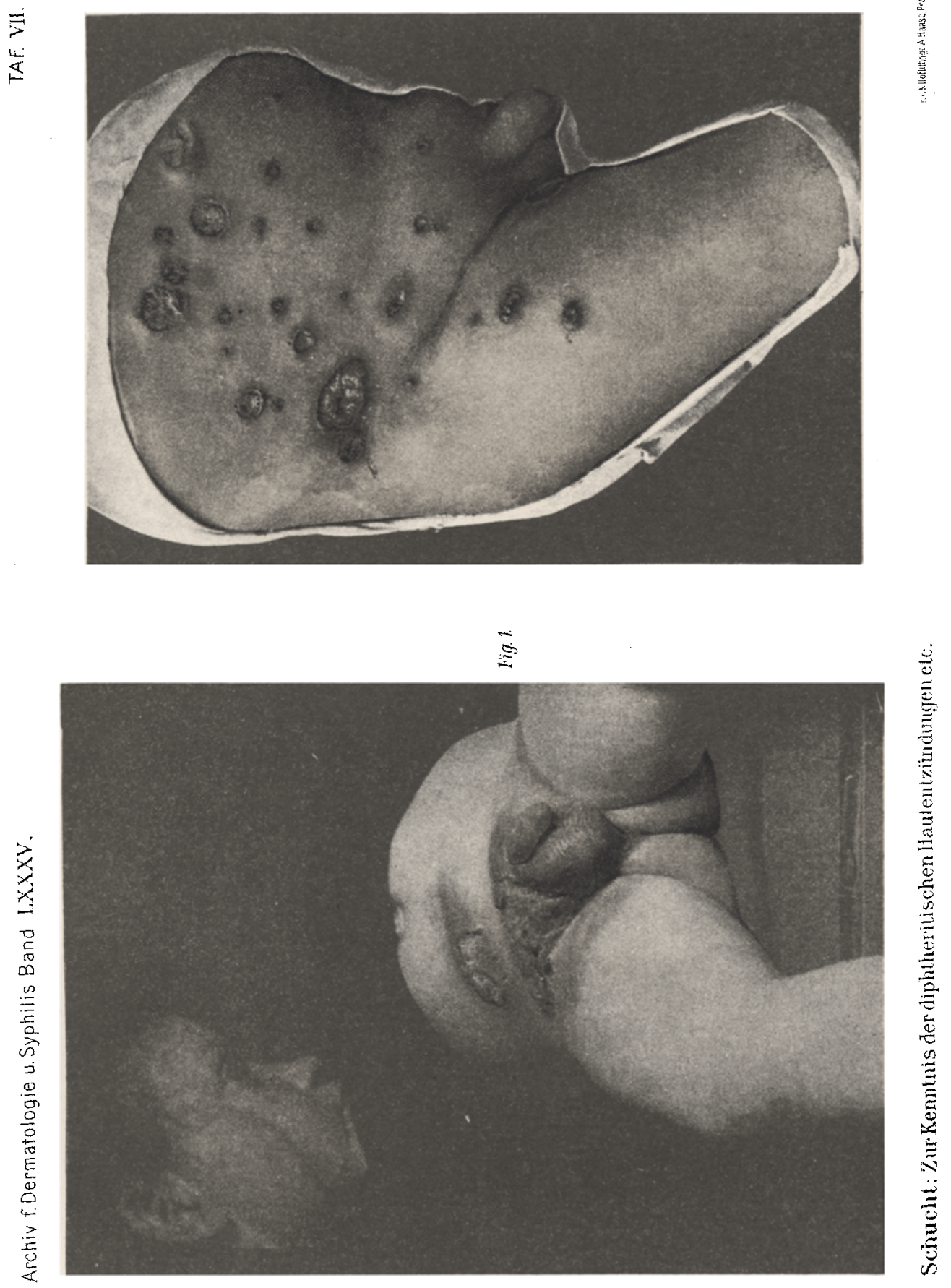